\title{
Characterazitions and Applications of Semistrictly Prequasi-invex Functions
}

\author{
Zaiyun Peng \\ Institute of Mathematics, College of Science \\ Chongqing JiaoTong University
}

66 Xue Fu Da Dao Street, Nan Ping, Chongqing 400074, China

E-mail: pengzaiyun@126.com.

Dacheng Wang, Yawei Liu, Zhi Lin

Institute of Mathematics, College of Science, Chongqing JiaoTong University

66 Xue Fu Da Dao Street, Nan Ping, Chongqing 400074, China

\begin{abstract}
A new type of generalized convex functions, termed semistrictly prequasi-invex functions, is further discussed in this paper. A sufficient condition and an impotant application of semistrictly prequasi-invex functions are obtained.
\end{abstract}

Keywords: Prequasi-invex functions, Semistrictly prequasi-invex functions, Applications

MSC(2000): 90C26,26B25 CLC number: O174.13

\section{Introduction}

Convexity and generalized convexity play a central role in mathematical economics and optimization theories. Therefore, the research on convexity or generalized convexity becomes one of the most important aspects in mathematical programming. A significant generalization of convex functions termed preinvex functions was introduced by (Weir,1998, p.29). Yang and Li obtained some properties of preinvex function in (Yang,2001, p.256).Yang also discussed the relationships among convexity, semistrictly convexity and strictly convexity in (Yang,1994, p.15). Recently, in (Yang,2001, p.645). Yang esterblished characterazitions of prequasi-invex functions and semistrictly prequasi-invex functions. In this paper, the relationship of prequasi-invex and semistrictly prequasi-invex function are further discussed, we obtain a new result that if a prequasi-invex function satisfies intermediate-point weakly semistrict prequasi-invexity then it is also a semistrictly prequasi-invex function. Then an impotant application of semistrictly prequasi-invex functions is obtained..

We give first the following definitions .

Definition 1.1 (Weir,1998, p.29) A set $K \subseteq R^{n}$ is said to be invex if there exist a vector function $\eta: R^{n} \times R^{n} \rightarrow R^{n}$, such that

$\forall x, y \in K, \forall \lambda \in[0,1] \Rightarrow y+\lambda \eta(x, y) \in K$.

Definition 1.2 (Yang,2001, p.645). Let $K \subseteq R^{n}$ be an invex set with respect to $\eta: R^{n} \times R^{n} \rightarrow R^{n}$. Let $f: K \rightarrow R$. We say that $f$ is prequasi-invex on $K$, if $\forall x, y \in K, \lambda \in[0,1]$,

$f(y+\lambda \eta(x, y)) \leq \max \{f(x), f(y)\}$.

Definition 1.3 (Yang,2001, p.645). Let $K \subseteq R^{n}$ be an invex set with respect to $\eta: R^{n} \times R^{n} \rightarrow R^{n}$. Let $f: K \rightarrow R$. We say that $f$ is semistrictly prequasi-invex on $K$, if $\forall \lambda \in(0,1) \forall x, y \in K, f(x) \neq f(y)$,

$f(y+\lambda \eta(x, y))<\max \{f(x), f(y)\}$.

Condition C. Let $\eta: R^{n} \times R^{n} \rightarrow R^{n}$. We say that the function $\eta$ satisfies Condition $\mathrm{C}$ if, for any $x, y \in K$, for any $\lambda \in[0,1]$,

$\eta(y, y+\lambda \eta(x, y))=-\lambda \eta(x, y)$, 
$\eta(x, y+\lambda \eta(x, y))=(1-\lambda) \eta(x, y)$.

Example1.1:Let

$$
\eta(x, y)=\left\{\begin{array}{l}
x-y,(x \geq 0, y \geq 0) \\
x-y,(x<0, y<0) \\
-2-y,(x>0, y \leq 0) \\
2-y,(x \leq 0, y>0)
\end{array}\right.
$$

By Example 2.2 in (Yang,2003, p.607),we know that $\eta$ satisfies the Condition C. Another example that $\eta$ satisfies the Condition C may refer Example 2.4 in (Yang,1995, p.901).

\section{Main Rerults}

In (Yang,2001, p.256), the authors have discussed the preinvex functions and semistrictly preinvex functions.In this section, we will discuss the properties of semistrictly prequasi-invex functions. At first, we will get a new result as follows.

Theorem 2.1 Let $K \subseteq R^{n}$ be an invex set with respect to $\eta: R^{n} \times R^{n} \rightarrow R^{n}$ which satisfies Condition C. Let $f: K \rightarrow R$, be a prequasi-invex function for the same $\eta$, and if for each pair of $x, y \in K, f(x) \neq f(y)$ there exists an $\alpha \in(0,1)$, such that

$$
f(y+\alpha \eta(x, y)<\max \{f(x), f(y)\}
$$

Then $f$ is semistrictly preqausi-invex on $K$ with respect to $\eta$.

Proof. By contradiction, we assume that $f$ is not semistrictly preqausi-invex on $K$. Then there exis $x, y \in K, f(x) \neq f(y), \lambda \in(0,1)$ such that

$$
f(y+\lambda \eta(x, y)) \geq \max \{f(x), f(y)\} .
$$

Let $z=y+\lambda \eta(x, y)$. Since $f$ is prequasi-invex, we have

$$
f(z)=f(y+\lambda \eta(x, y)) \leq \max \{f(x), f(y)\}
$$

The above two inequalities imply that

$$
f(z)=\max \{f(x), f(y)\}
$$

We note that the pair $x, z \in K$ and $y, z \in K$, such that $f(x) \neq f(z), f(z) \neq f(y)$.From inequality (1), there exists an $\beta_{1}, \beta_{2} \in(0,1)$, such that

$$
\begin{aligned}
& f\left(z+\beta_{1} \eta(x, z)\right)<\max \{f(x), f(z)\} \\
& f\left(y+\beta_{2} \eta(z, y)\right)<\max \{f(z), f(y)\}
\end{aligned}
$$

Denote

$$
\bar{x}=z+\beta_{1} \eta(x, z), \quad \bar{y}=y+\beta_{2} \eta(z, y)
$$

Then , from(4)--(6), we have

$$
f(z)>f(\bar{x}), \quad f(z)>f(\bar{y})
$$

And from condition $\mathrm{C}$,

$$
\begin{gathered}
\bar{x}=z+\beta_{1} \eta(x, z)=y+\lambda \eta(x, y)+\beta_{1} \eta(x, y+\lambda \eta(x, y)) \\
=y+\left[\lambda+\beta_{1}(1-\lambda)\right] \eta(x, y) \\
\bar{y}=y+\beta_{2} \eta(z, y)=y+\beta_{2} \eta(y+\lambda \eta(x, y), y) \\
=y+\beta_{2} \eta(y+\lambda \eta(x, y), y+\lambda \eta(x, y)-\lambda \eta(x, y)) \\
=y+\beta_{2} \eta(y+\lambda \eta(x, y), y+\lambda \eta(x, y)+\eta(y, y+\lambda \eta(x, y))) \\
=y+\beta_{2} \lambda \eta(x, y)
\end{gathered}
$$


Let $u_{1}=\lambda+\beta_{1}(1-\lambda), \quad u_{2}=\beta_{2} \lambda, \quad u=\left(\lambda-u_{2}\right) /\left(u_{1}-u_{2}\right)$.

It is easy to verify that $u_{1}, u_{2}, u \in(0,1)$.Again from Condition $\mathrm{C}$,

$$
\begin{aligned}
& \bar{y}+u \eta(\bar{x}, \bar{y})=y+u_{2} \eta(x, y)+u \eta\left(y+u_{1} \eta(x, y), y+u_{2} \eta(x, y)\right) \\
= & y+u_{2} \eta(x, y)+u \eta\left(y+u_{1} \eta(x, y), y+u_{1} \eta(x, y)+\left(u_{2}-u_{1}\right) \eta(x, y)\right) \\
= & \left.y+u_{2} \eta(x, y)-u\left[\left(u_{2}-u_{1}\right) /\left(1-u_{1}\right)\right] \eta\left(x, y+u_{1} \eta(x, y)\right)\right) \\
= & y+\left[u_{2}-u\left(u_{2}-u_{1}\right)\right] \eta(x, y)=y+\lambda \eta(x, y)=z
\end{aligned}
$$

Since $f$ is prequasi-invex function on $K$, this implies

$$
f(z) \leq \max \{f(\bar{x}), f(\bar{y})\}
$$

Which contradicts (7). This completes the proof.

Remark1. From the Theorem2.1, we obtain a sufficient condition of semistrictly prequasi-invex functions, and we also see that a uniform $\alpha \in(0,1)$ is not necessary for inequality (1) in Theorem 2.1.

Let the problem of minimum $f(x)$ subject to $x \in K$ be denoted by (P). Now, we can discuss an application of semistrictly prequasi-invex functions to the problem $(\mathrm{P})$.

Theorem2.2 Let $K \subseteq R^{n}$ be a nonempty invex set with respect to $\eta: R^{n} \times R^{n} \rightarrow R^{n}$ and $f: K \rightarrow R$ be a semistrictly prequasi-invex function with respect to $\eta$.If $\bar{x}$ is a local minimum to the problem (P), then $\bar{x}$ is a global one.

Proof. If $\bar{x}$ is a local minimum to the problem (P), then there exists a neighborhood $U \in R^{n}$ with $\bar{x} \in U$ and

$$
f(\bar{x}) \leq f(x), \forall x \in K \cap U
$$

Assume that $\bar{x}$ is not a global one of $(P)$,then there exists $\hat{x} \in K$ with

$$
f(\hat{x}) \leq f(\bar{x})
$$

Since $K \subseteq R^{n}$ be a nonempty invex set with respect to $\eta: R^{n} \times R^{n} \rightarrow R^{n}$ and $f: K \rightarrow R$ be a semistrictly prequasi-invex function, then $\forall \lambda \in(0,1)$

$f(\bar{x}+\lambda \eta(\hat{x}, \bar{x}))<\max \{f(\hat{x}), f(\bar{x})\}=f(\bar{x})$

i.e.,for any $\forall \lambda \in(0,1)$, we have

$$
f(\bar{x}+\lambda \eta(\hat{x}, \bar{x}))<f(\bar{x})
$$

$\lim _{\lambda \rightarrow 0}(\bar{x}+\lambda \eta(\hat{x}, \bar{x}))=\bar{x} \quad, \quad$ so there exists $\quad$ a $\quad \delta$ and $0<\delta<1$ and for all $\lambda \in(0, \delta)$ with $\bar{x}+\lambda \eta(\hat{x}, \bar{x}) \in K \cap U$. Then (10) is contrary to (8). This completes the proof.

Remark 2. From Theorem 2.2, we can conclude that the class of semistrictly prequasi-invex functions constitutes an important class of generalized convex functions in mathematical programming.

\section{Acknowledgment}

This study is supported by Supported by Natural Science Foundation Project of CQ CSTC(2008BB0346)and Youth Science Project Research Foundation of Chongqing Jiao Tong University.

The authors also would like to thank two anonymous referees for their valuable comments and suggestions, which helped to improve the paper.

\section{References}

Weir, T and Mond, B. Preinvex Functions in Multiple Objective Optimization.J Math Anal Appl.1988,136:29-38.

Yang, X, M and Li, D. On Properties of Preinvex Functions.J Math Anal Appl 2001,256:229-241.

Yang, X, M. Semistrictly convex Functions.Opsearch,1994,31(1):15-27.

Yang, X, M and Li, D. Semistrictly Preinvex Functions.J Math Anal Appl 2001,258:287-308. 
Mohan, S, R and Neogy, S, K. On Invex sets and Preinvex Functions.J Math Anal Appl,1995,189:901-908.

Yang, X, M,Yang, X, Q and Teo, K, L. Characterazition and Applications of prequasi-invex functions, Journal of optimization theory and Applications 2001,110(3): 645-668.

Yang, X, M,Yang, X, Q and Teo, K, L.Generalized invexity and invariant monotonicicy.J Optim Theory Appl. 2003,117:607-625.

Luo, H. L. and Peng, Z. Y. General Convergence analysis for generalized n-step projection methods and applications to a kind of Variational Inequality problems, J.of Southwest University(Natural Science),2007 (32)1-4.

Peng, Z. Y. and Luo, H. L. The Three-step Projection Method in Generalized Analysis and Its Application to Variational Inequality Problems. Journal of Sichuan Normal University(Natural Science edition),2007.p681-683.

Peng, Z. Y. and Chen, Guo. Remarks on Prequasi-invex Functions. Journal of China Three Gorges University (Natural Science edition),2007.p70-72.

Peng, Z. Y. and Long, X.J. A Class of Random F-complementarity Problems in Hilbert Spaces, Advanced in Nonlinear Variational Inequalities, 11(2008)51--59.

Peng, Z. Y. and Luo, H. L. Technical Note on Characterrizations of Strongly Preinvex Functions. Journal of Chongqing Normal University(Natural Science edition),23(2006)36--39.(in chinese). 